\title{
Fokusakzente als rhetorische Hervorhebungsmarker in der gesprochenen Wissenschaftssprache. Eine deutsch-polnische vergleichende Signaluntersuchung
}

Das Ziel der folgenden Überlegungen besteht in der exemplarischen Darstellung sowie einer anfänglichen Systematisierung prosodischer Fokusakzente mit rhetorischer Funktion in gesprochenen Wissenschaftstexten. Dazu sind vorab einige einführende Bemerkungen methodologischer und terminologischer Natur unentbehrlich. Um das eigentliche Ziel folgender Überlegungen, d.h. die exemplarische Darstellung prosodischer Fokusakzente mit rhetorischer Funktion in gesprochenen Wissenschaftstexten und den Vorversuch diese Erscheinung einigermaßen zu systematisieren, effektiver zu realisieren, sind einige Einführungsbemerkungen methodologischer und terminologischer Natur an dieser Stelle unentbehrlich.

Es ist wohl für alle, die sich mit der Phonetik beschäftigen klar, dass diese linguistische Disziplin relativ eindeutig die lautliche Manifestation der Sprache beschreibt, solange man auf den sogenannten segmentalen und intersegmentalen Ebenen des phonetischen Ausdrucks bleibt, d.h.: solange man die Artikulation beziehungsweise die Koartikulation der Segmente, also der Sprachlaute untersucht (vgl. u.a. Tworek 2012:45-47). Geht man über diese Ebenen hinaus, versucht man oberhalb der Segmentsequenzen das Lautliche zu analysieren, wird man zahlreichen diversen Problemen, in erster Linie gerade methodologisch-terminologischer Natur, ausgesetzt. Ihre Quelle ist, dass die zu analysierte Materie vielschichtig ist, aus mehreren unterschiedlich hierarchisierten Phänomenen besteht, sich leicht der im Grunde genommen objektiven Artikulation entzieht und die physikalische Realität mit physikalisch nur schwer definierbaren akustischen Parametern zu erfassen verlangt, was letztendlich auf der perzeptorischen Seite bestenfalls von Wahrnehmungseindrücken sprechen lässt. Dies provoziert wiede- 
rum das terminologische Chaos - also etwas, was für die Sprachwissenschaft im Allgemeinen charakteristisch ist und ihre Zugehörigkeit zu humanistischen Wissenschaftsdisziplinen zu bestätigen vermag. Dennoch ist das Chaos im Bereich der Phonetik (auch der Phonologie, auch der Phonodidaktik) besonders irritierend. Anhand maßgebender Fachliteratur eindeutig zu sagen, was beispielsweise die Akzentuierung, die Betonung, die Intonation, die Prosodie sind, ist kaum möglich. ${ }^{1}$ Das terminologische Chaos wird noch zusätzlich dadurch vertieft, dass solche Termini in vielen allgemeinlinguistischen - also nicht phonetischen oder phonologischen - Bearbeitungen gern verwendet werden und Spezialisten in anderen linguistischen Subdisziplinen sie eher intuitiv (was übrigens von vorne herein kein Vorwurf sein darf) verstehen. Das bewahrtt sie aber nicht davor, ihre methodologischen Defizite zu kompensieren, indem sie zum Beispiel der sogenannten Intonation (was auch immer sie bedeuten sollte) bestimmte syntaktisch-semantische Funktionen zuschreiben. Es wird behauptet (dazu mehr Tworek 2013), dass es beispielsweise eine Subjekt- oder Objektintonation gibt, oder dass Phorika bzw. Augmentativa ebenfalls intonatorisch erkennbar sind, obwohl es bereits als bewiesen gilt, dass dies nicht der Fall ist. ${ }^{2}$ Erwähnenswert ist das oben Gesagte nur deswegen, weil man gerade in solchen Situationen am häufigsten mit den in der Regel syntaktisch unbelasteten Fokusakzenten zu tun hat.

Was sind diese Fokusakzente? Sie sind ja Akzente - dienen also zur Hervorhebung. Phonetisch hervorgehoben wird auf zwei Ebenen des lautlichen Ausdrucks der Sprache: auf der sogenannten suprasegmentalen und auf der sogenannten prosodischen. Terminologische - aber manchmal auch inhaltliche - Aussonderung der beiden Ebenen liegt in der Fachliteratur nicht immer vor. Dies hängt weitgehend damit zusammen, ob man diese Erscheinungen aus der sprech- oder sprachwissenschaftlicher Perspektive zu bestimmen versucht. In der erstgenannten differenziert man sie im Prinzip nicht aus, da sprechwissenschaftlich nicht die Strukturen der Sprache, sondern ihre Performanz in Sprechprozessen analysiert werden. ${ }^{3}$ In der sprachwissenschaftlichen Perspektive wird dagegen diese Performanz in Bezug auf Sprachstrukturen eruiert, auch wenn in dieser Hinsicht keine vollständige Systematizität zu erwarten ist. Da die methodologische Grundlage folgender Überlegungen sprachwissenschaftliche Sichtweise bildet, differenzieren wir die beiden Ebenen der Phonetik wie folgt ${ }^{4}$ : Zum Bereich der suprasegmentalen Phonetik gehören diejenigen Phänomene, die punktuell markierbar sind

\footnotetext{
1 Symptomatisch ist eine Tabelle, mit der Emiko Inozuka ihre Monographie „Grundzüge der Intonation. Definition und Methodologie in deutschen Intonationsmodellen“ (2003) abschließt, in der diesbezügliche Termini und ihre Designate bei mehreren Autoren zusammengestellt werden und in der so gut wie keine Übereinstimmung herrscht.

2 Mehr dazu vgl. u.a. Tworek (2013).

3 Vgl. u.a. Kreuz/Mundwiler (i.d.B.). Dazu mehr u.a. Fiukowski ( $\left.{ }^{8} 2010\right)$, Bose et al. (2013).

${ }^{4}$ Vgl. Tworek 2012:209ff. Mehr zu diesen theoretischen Ausgangspunkten u.a. Lehiste (1970), Canepari (2007).
} 
und zur Hierarchisierung der segmentalen Einheiten - also der Sprachlaute - dienen. Und als prosodisch werden diejenigen Phänomene betrachtet, die als linear zu erfassen sind, sich grundsätzlich auf Einheiten erstrecken, die länger als ein Segment sind und damit den Rhythmus und die Melodie des Ausdrucks ausmachen. Suprasegmental wird also innerhalb eines Wortes (phonetisch gesehen eines Mehrsilbers) hervorgehoben, prosodisch wird dagegen innerhalb eines Ausdrucks (in Form eines Satzes, einer längeren Phrase usw.) hervorgehoben. Unterschiedlich sind die Bezugsgrößen der Hervorhebung. Rein physikalisch spricht man gewöhnlich von drei Hauptparametern der Hervorhebung in Form von Akzentuierung/Betonung. Es sind Grundtonfrequenz, Quantität und Intensität. Begleitet können sie noch von weiteren Phänomenen werden, wie zum Beispiel Lautstärke, Sprechtempo oder auch übertriebene Realisierungen auf der (inter)segmentalen Ebene (Artikulation). Die akustisch-phonetischen Messungen beweisen, dass der primäre (auch aus der perzeptiven Perspektive) und einzig obligatorische Parameter der suprasegmentalen Wortbetonung die Änderung der Grundtonfrequenz (akustisch sind das die sog. Nullformanten) ist. Dies führt übrigens dazu, dass tatsächlich nicht die Silben betont sind, sondern nur ein Segment, das zum sog. Silbengipfelträger wird und unbedingt stimmhaft sein muss, denn die stimmlosen Sprachlaute über keinen Grundton verfügen. Und gerade Fokusakzente, die des Weiteren als prosodische Phänomene zu betrachten sind, werden nämlich viel stärker durch die sonstigen Faktoren determiniert, d.h. durch Änderungen der Lautstärke oder des Sprechtempos (nicht nur ihre Intensivierung, auch ihre Senkung bzw. Minimierung), durch die Intensität (verstanden als zusätzliche exspiratorische Kraft der Artikulation) und die Quantität (Verlängerung, Ausdehnung eines Segments, übrigens nicht nur eines Vokals). Die Varianzen in der Lautstärke oder im Sprechtempo erstrecken sich nicht selten auf die nicht fokussiert akzentuierten Elemente, sondern sogar auf ganze Ausdrücke. Dazu kommen noch individuelle Kreativität und sprechmotorische Fähigkeiten eines Sprechers. Sie eröffnen Freiräume für unterschiedlich gezielte Modifizierungen der Stimme, was besonders die künstlerischen Äußerungen kennzeichnet. Fokusakzente sind schließlich nicht die einzigen prosodischen Hervorhebungsmarker, mit denen die komplexe lineare rhythmisch-melodische Gestaltung des gesamten Ausdrucks manifestiert wird. Die zwei weiteren wichtigsten Phänomene sind Pausen und Tonführung. Daher also sind Fokusakzente nicht einseitig zu definieren und müssen eher funktional und nicht physikalisch bestimmt werden. Funktionen und Ziele der Fokusakzentsetzung hängen logischerweise mit - abgesehen von vielen weiteren Determinanten - der Art des gesprochenen Textes, seinen kommunikativen aber auch performativen Aufgaben zusammen.

Diese Annahme baut eine Brücke, die uns des Weiteren direkt zur Rhetorik führt. Wir wollen die Rhetorik an dieser Stelle weder systematisch definieren noch grundsätzlich beschreiben, unter anderen deswegen, da dies in zahlreichen Texten in diesem Band, in denen sie zum wichtigsten Untersuchungsobjekt geworden 
sind, ausführlich gemacht wird. Für die weiteren Überlegungen ist es ausreichend relevant, wenn angenommen wird, dass Rhetorik funktional gesehen als eine komplexe Vorgehensweise verstanden wird, die zur Optimierung der Übertragung und der Wahrnehmung der kommunikativen Absicht in einem Redefluss beizutragen hat oder kurz und gehoben als die Kunst gut und wirkungsvoll zu reden, betrachtet wird. Dann unterliegt es keinem Zweifel, dass es auch phonetische - darunter auch prosodische - Mittel gibt, die helfen können, diese Ziele zu erreichen. ${ }^{5}$ Leider hat man auch in diesem Bereich ab und zu mit unterschiedlichen pseudointuitiv gefassten Stereotypen zu tun. So liest man beispielsweise in einigen Handbüchern der Rhetorik (vgl. z.B. Allnach/Rusch 1995) unter vielen sinnvollen Ratschlägen auch von solchen, nach denen zum Beispiel die häufige Verwendung der gerundeten Vokale positive Emotionen beim Hörer effektiver wecken solle als die der gespreizten. Auch wenn das in Bezug auf bestimmte auBersprachliche Empfindungsmuster absoluter akustischer Werte teilweise der Fall sein mag, ist mit ihrer Übertragung auf die sprachlich realisierten Kommunikate nicht zu rechnen. Der deutsche Satz Warum lügst du so oft ist voll von gerundeten Vokalen und ein anderer Ich liebe dich sehr besteht ausschließlich aus gespreizten Vokalen und das lässt sich ja nicht ändern!

Es wird auch auf der anderen Seite behauptet ${ }^{6}$, dass der Ausdruck von negativen Emotionen im Polnischen durch die Verwendung von Wörtern mit dem [r]-Laut intensiviert wird - wohl deswegen, dass das bekannteste polnische Schimpfwort kurwa ein solches [r] im Inlaut hat. Dass das aber nicht ernst zu nehmen ist, zeigen die ganzen Listen von Lexemen, die trotz der [r]-Absenz sehr effektiv negativ geladene Emotionen semantisieren können und wenn die anderen slawischen Sprachen, in denen wohl noch lieber geschimpft wird, berücksichtigt werden (die östlichen - Russisch, Ukrainisch; oder die südlichen - Kroatisch, Serbisch), stellt sich heraus, dass die häufigsten Schimpfwörter dort doch [r]-los sind. Die Artikulation des [r] kann zweifellos aus sprechmotorischen Gründen zu irgendwelchen Hervorhebungen oder zur gewissen Wahrnehmungsintensivierung dienen, kann aber keineswegs als Marker artiger bzw. vulgärer Inhalte interpretiert werden.

So resultieren Gesagten resultieren bestimmte Prämissen für die Untersuchung der prosodischen Fokusakzente. Um diese Untersuchung möglichst zu systematisieren, müssen zwei grundsätzliche Fragen gestellt und damit zwei unterschiedliche Erscheinungen in Betracht gezogen werden. Zum Einen lautet die Frage - was?, zum Anderen lautet sie - wie?

In jeder Antwort auf die erstere Frage muss gezeigt werden, was fokussierend akzentuiert wird, was in einem inhaltlich abgrenzbaren Redeflussfragment

5 Auch der absichtliche Verzicht auf derartige Mittel kann als Extremfall der rhetorischen Gestaltung eines gesprochenen Textes gelten. Beispiel dafür sind unter anderen die sog. Thronreden der britischen Königin.

6 Vgl. Sikorski (2003). 
hervorgehoben wird. Es geht dabei um diejenigen Einheiten, die als sprachstrukturelle Bestandteile einer gegebenen Äußerung (bzw. Äußerungsteils) dienen. Theoretisch gesehen können das sein: bestimmte Einzelsegmente (wo nach artikulatorischen Besonderheiten - Modifizierungen, Intensivierungen, Lenisierungen, Tilgungen, Hinzufügungen, Ersetzungen usw. - zu suchen ist); einige Segmentsequenzen (wo intersegmentale koartikulatorisch-assimilatorische Prozesse aktiv sind); Segmentsequenzen, die eine sprachstrukturell höhere Einheit in Form von informativ belasteten Morphemen bilden; Wörter (wenn sie betonbare Mehrsilber sind, ist auch nach eventuellen Verschiebungen der in den beiden hier analysierten Sprachen festen vokalischen Silbengipfelträger der suprasegmentalen Wortbetonung zu suchen) und schließlich Phrasen ${ }^{7}$, allerdings nur diejenigen, in denen zwischen ihren Bestandteilen keine Pausen signifikant aktiviert werden. Außer Acht lassen wir dagegen diejenigen Situationen, wo die Fokussierung sich auf längere Einheiten erstreckt (z.B. überlange Phrasen, Sätze, längere Äußerungsfragmente). Solche Erscheinungen sind auch möglich und kommen vereinzelt vor. Sie sind jedoch erst aus einer Perspektive sichtbar, die grundsätzlich eine ganze Aussage als Bezugshintergrund umfasst.

Und die angedeutete wie-Frage, betrifft die materielle, physikalische Form mit der die Fokusakzente zum phonetischen Ausdruck gebracht werden. Diese Fokussierungsmarker wurden bisher bereits genannt (artikulatorische Modifikationen, Intensität, Lautstärke, Sprechtempo usw.). Sie sind allerdings alle Marker, die vom Empfänger wahrnehmbar sein müssen, sonst wäre ihre Auswirkung so gut wie keine gewesen. Als eine theoretische Voraussetzung - wenn auch mit keiner Realisierungsgarantie - ist in diesem Zusammenhang auch die Erwartung zu nennen, dass gerade die Hervorhebungsabsicht des Sprechers wahrgenommen werden muss. Die Effektivität einer solchen Wahrnehmung wächst, wenn bestimmte Fokusakzente kongruent eingesetzt werden. Dabei handelt es sich um eine Kongruenz ${ }^{8}$ auf mindestens drei Stufen:

Erstens - internprosodische Kongruenz: Bei der Vielfalt der angesprochenen prosodischen Fokussierungsmarker wäre es günstig, wenn mit ihnen nicht kontraproduktiv agiert wird.

Zweitens - externprosodische Kongruenz: Es wäre effektiv, wenn die Fokusakzente mit anderen prosodischen Phänomenen ein kompaktes Gesamtbild ausmachen würden. In aller ersten Linie geht es um die bestimmte Kongruenz der Fokusakzente mit Pausen.

Drittens - außerprosodische Kongruenz: Es geht um die Kongruenz der Fokusakzente mit einzelnen Bestandteilen kognitiv realisierbarer Sprachstrukturen,

\footnotetext{
${ }^{7}$ All die aufgelisteten fokussierbaren sprachstrukturellen Einheiten lassen sich entsprechend auch lautorganisatorisch bestimmen, worauf jedoch aus methodologischen Gründen an dieser Stelle verzichtet wird. Dazu mehr u.a. bei Tworek (2012:14).

${ }^{8}$ Nicht zu vergessen sind auch diejenigen Situationen, wo die Kongruenz absichtlich unterbrochen wird um gewisse Kontrasteffekte zu erzielen.
} 
also vor allem mit ihrer Semantik, aber auch mit der Syntax (ggf. auch mit der Morphologie).

Die Realisierung von Fokusakzenten ist natürlich kein Ziel an sich, vielmehr muss sie einer übergeordneten, zumeist kommunikativen Funktion dienen.

Die anscheinend primäre Funktion in diesem Zusammenhang könnte die informative Unterstreichung eines relativ kleinen Textausschnitts sein. $\mathrm{Ob}$ es in der Tat immer so ist, bleibt fraglich. Besonders wenn prosodische Struktur einer Äußerung absichtlich gestaltet wird. Dann kann es zu anderen Motivationen kommen: schöne melodisch-rhythmische Gestaltung des Textes und Zufriedenheit mit dem Klang eigener Stimme oder verschiedenartige Manipulationen oder aber unbeabsichtigte Realisierungen, die beispielsweise Folgen äußerlicher Störungen beliebiger Natur und Art sein können. Schließlich ist an dieser Stelle noch eine gewisse Inkompetenz des Sprechers zu erwähnen, der einfach gesagt - aus sehr unterschiedlichen Gründen ${ }^{9}$ und in sehr unterschiedlichen Formen - prosodisch falsch agieren kann.

\section{Untersuchung}

Das folgende Untersuchungsmaterial stammt aus dem „GeWiss“-Korpus, das im Rahmen eines internationalen Projekts zur Erforschung der gesprochenen deutschen Wissenschaftssprache im Vergleich mit dem Englischen und Polnischen am Herder-Institut in Leipzig in den Jahren 2009-2013 gesammelt worden ist. ${ }^{10}$ Die an dieser Stelle angeführten Beispiele - wegen des Charakters einer Signaluntersuchung bilden sie nur eine Art Exemplifikation und sind keineswegs quantitativ zu bewerten - werden aus sogenannten im „GeWiss“-Korpus archivierten Expertenvorträgen exzerpiert, einer Textsorte also, die meistens ursprünglich als geschriebener Text vorliegt und erst dann gesprochen manifestiert wird. Dennoch (oder gerade deswegen) werden solche Vorträge gewissermaßen rhetorisch gestaltet. Zum Instrumentarium rhetorischer Gestaltung dieser Texte gehören auch phonetische und darunter prosodische Mittel und unter ihnen gerade Fokusakzente. Es ist symptomatisch, dass sich gleich nach 5-6 Minuten der beiden Quellenvorträge etwa 20 solche Fokusakzente wahrnehmen lassen. Im Folgenden werden je zehn deutsche und polnische Beispiele diesbezüglich analysiert. Die beiden Sprecher - ein deutscher und ein polnischer Muttersprachler - sind männlich, über 30 Jahre alt, beide sind Textlinguisten und hier sprechen sie dialektfrei.

\footnotetext{
${ }^{9}$ Dies ist nicht selten u.a. beim Sprechen in einer Fremdsprache der Fall, wenn die nötige Sicherheit und Natürlichkeit gewissermaßen nachlassen. Und gerade diese Natürlichkeit ist für den geschickten (auch wenn er kontrolliert wird) Umgang mit prosodischen Mitteln eine unvermeidbare Voraussetzung. Dazu vgl. u.a. Reinke (2008).

${ }^{10}$ Mehr dazu: https://gewiss.uni-leipzig.de. Vgl. auch Rogozińska (i.d.B.).
} 
Den zehn deutschen (DT) Beispielen folgen direkt die polnischen (PL), alle nummeriert. Die fokussierten Einheiten werden mit Fettdruck markiert. In Klammern werden jeweilige lautliche Phänomene notiert: LS - Lautstärke, ST Sprechtempo, AI - Artikulationsintensität. Plus- und Minus-Zeichen deuten auf Erhöhung bzw. Senkung der Realisierungskapazität gegebener Phänomene (Doppelzeichen bedeutet extreme Realisierung). Punkte (ggf. ihre Zahl) in Klammern markieren Pausen (ggf. ihre Länge). In eckigen Klammern gibt es Notationen zu (supra)segmentalen Merkmalen (nach der API-Transkription). Unterstrichene Fragmente sind mit in die Fokussierung eines Nachbarelements involviert. Kurze Kommentare schließen die Anführung jedes Beispiels ab.

\section{DT 1}

um das circa (..) ja[a::] (..) zwanzig graduierungsarbeiten unterschiedlicher thematik zentriert ist

Kommentar: Interpausale Stellung mit deutlicher artikulatorischer Ausdehnung des vokalischen Segments. Kein direkter Bezug auf die Semantik.

\section{DT 2}

da es sich ... sehr häufig erkenntnistheoretiker und philosophen zu wort gemeldet haben die äh gerade wie vireleau der französische äh philosoph sagten hier verschwindet (ST -) etwas (ST - -)

Kommentar: Bereits bei verschwindet sinkt das Sprechtempo und etwas wird extrem langsam ausgesprochen. Keine Pausen. Bezug auf die Semantik (ansatzweise) vorhanden.

\section{DT 3}

man sagt sogar die kommunikation sei (...) ortlos (LS -)

Kommentar: Postpausale Stellung, leise Aussprache mit Tonmustersenkung. Bezug auf die Semantik vorhanden.

\section{DT 4}

die reaktion ... auf diese metaphysische position ... ist natürlich zu sagen (..) nein $(\mathrm{ST}++/ \mathrm{LS}+)($.$) das ist nicht der fall (\mathrm{ST}+/ \mathrm{LS}=)$

Kommentar: Interpausale Stellung mit Sprechtempo- und Lautstärkeerhöhung, die zum Teil in der nächsten Phrase noch wahrnehmbar sind. Bezug auf die Semantik vorhanden. 
DT 5

die kommunikation äh und (..) $\boldsymbol{r}[\mathrm{R}::]$ aum $(\mathrm{ST}-)$ genauso wie die zeit $(\mathrm{ST}+)$

Kommentar: Postpausale Stellung mit kontrastierender Sprechtemposenkung und auffallend ausgedehnte Aussprache des /r/-Segments mit uvularen Vibrationen. ${ }^{11}$ Bezug auf die Semantik vorhanden.

DT 6

das ist nämlich die ak['ak]tuelle (AI + / ST ++) (..) untersuchungsgröße der fachsprachenforschung

Kommentar: Präpausale Stellung mit Sprechtempobeschleunigung. Verschiebung der suprasegmentalen Betonung (begleitet durch Erhöhung der artikulatorischen Intensität) auf den anlautenden Vokal. Bezug auf die Semantik vorhanden.

DT 7

diesen raumdiskurs er ist noch $(\mathrm{AI}+/ \mathrm{ST}++)$ intensiver als bei uns

Kommentar: Sprechtempo- und Intensitätserhöhung. Kongruenz mit der semantischen Intensivierungsfunktion des noch. Keine Pausen.

DT 8

es gibt eine un['ত̃::n:]wahrscheinliche $(\mathrm{ST}++)$ vielfalt

Kommentar: Sprechtempobeschleunigung mit artikulatorischer Verlängerung beider anlautender Segmente und auffallender regressiver Nasalierung des Vokals. Keine Pausen. Bezug auf die Semantik vorhanden.

DT 9

wenn ich nicht die grund ['v:n::t] $(\mathrm{AI}+/ \mathrm{ST}--)$ lage $(\mathrm{ST}++)$ für meine sichtweise

Kommentar: Artikulatorische Intensivierung bei grund gekoppelt mit Verlängerung der [ชn]-Segmentsequenz und Sprechtemposenkung kontrastiert mit extremer Sprechtempobeschleunigung bei lage. Keine Pausen. Bezug auf die Semantik vorhanden.

11 Die sonstigen nicht vokalisierten /r/-Konsonanten realisiert der Sprecher zwar auch uvular aber frikativ. 
DT 10

denn da $[\mathrm{a}::](\mathrm{LS}+)($.$) ist sehr viel angeschoben worden$

Kommentar: Extreme ${ }^{12}$ artikulatorische Ausdehnung des vokalischen Auslautssegments mit Lautstärkeerhöhung. Präpausale Stellung. Kein direkter Bezug auf die Semantik (bis auf phorische Referenz).

PL 1

temat problem który chciałem za(..)prezentować (ST -)

Kommentar: Intermediäre Pause mit Neuordnung der Wortbildung, langsames Sprechtempo (besonders bei $z a$ ). Fokussiert wird ein für die Äußerungssemantik wenig relevantes Wort.

PL 2

od kilku lat zajmuję się deklaracją misji przedsiębiorstw $(\mathrm{LS}+/ \mathrm{ST}++)$ (.) głównie przedsiębiorstw ( $\mathrm{LS}+/ \mathrm{ST}++$ )

Kommentar: Lautstärkeerhöhung und extreme Sprechtempobeschleunigung ${ }^{13}$ verstärkt durch die Wiederholung. Intermediäre Pause. Bezug auf die Semantik vorhanden.

PL3a

można napisać mono(AI + / LS + +)grafię deklaracji misji ...

Kommentar: Keine Pausen. Extreme Lautstärke- und Intensitätserhöhung bei mono minimiert die Wirkung der tatsächlichen normgerechten suprasegmentalen Betonung auf präfinalem [a]. Starke Hervorhebung der mono-Semantik.

PL 3b

... wybratem definicję najkr[r::]ótszą (LS +)

12 Nach dem perzeptiven Eindruck scheinen alle drei langen (und gespannten) Vokale im weiteren Teil der Äußerung kürzer als das Auslauts-[a] im $d a$.

13 Die Wortform przedsiębiorstw ist artikulatorisch ziemlich anspruchsvoll und sie zu beschleunigen ist eine artikulatorische Herausforderung. 
Kommentar: Keine Pausen. Lautstärkeerhöhung mit auffallend größerer Zahl (sechs) von apikalen Anschlägen ${ }^{14}$ bei der Artikulation des [r]-Segments. Bezug auf die Semantik ansatzweise vorhanden.

PL 4

I misja jest dokumentem (..) stra['a](LS + +)tegicznym (LS+ / ST +) (..) zarzadzania strategicznego

Kommentar: Sprechtempobeschleunigung und Lautstärkeerhöhung (besonders bei stra). Verschiebung der suprasegmentalen Betonung (Hauptbetonung auf dem Vokal der Anlautsilbe, auf dem normgerechten präfinalen [i] liegt nur noch eine schwache Nebenbetonung). Postpausale Stellung. Der Mangel an Postpause stört die Äußerungstopographie und damit ihre kommunikative Absicht, was ein textsortenspezifischer Fall unabsichtlicher Sprecherinkompetenz ist. Infolgedessen ist die semantische Hervorhebung nur begrenzt vorhanden.

PL 5

inni badacze sięgają siedmiu ... elementów deklaracji misji (.) i znowu (AI + + I $\mathrm{LS}+)$ (..) co ma się na deklaracje misji składać to ...

Kommentar: Interpausale Stellung mit Intensitäts- und Lautstärkeerhöhung. Fokussiert wird eine Phrase (im syntaktischen Sinne) mit wenig signifikanter Semantik.

PL 6

szereg funkcji ... nastęujaco (..) opisowo identyfikacyjna (..) czym jest firma (..) planistyczna (AI + / LS + / ST -) (..) ponieważ deklaracja misji daje pewna wizje

Kommentar: Komplexe Fokussierung mit drei Phänomenen (Intensität und Lautstärke wachsen, Sprechtempo sinkt) in interpausaler Stellung. Pausenverwendung (Präsenz, Absenz) in sonstigen Äußerungsteilen erschwert die Wahrnehmung inhaltlicher Zuordnung, dennoch ist die semantische Hervorhebung vorhanden.

\section{PL 7}

$w$ dwóch kierunkach (..) $\boldsymbol{k o}\left[{ }^{\prime} \mathrm{o}\right](\mathrm{AI}++)$ munikuje się (.) zarówno wewnątrz przedsiębiorstwa z pracownikami

14 In den meisten Fällen realisiert der Sprecher einen bis zwei von solchen apikalen Anschlägen. 
Kommentar: Interpausale Stellung mit deutlich erhöhter Artikulationsintensität, die eine Verschiebung der suprasegmentalen Hauptbetonung auf initialen Vokal

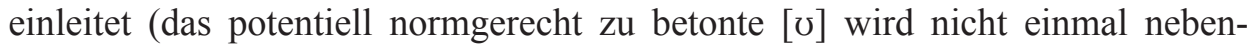
betont). Fokussiert wird eine Phrase (im syntaktischen Sinne) mit ansatzweise signifikanter Semantik.

PL 8

... (.) mimo $(\mathrm{AI}++/ \mathrm{ST}++) \underline{\text { wszystko }}(\mathrm{ST}+)$ zdaniem swalesa można mówić...

Kommentar: Postpausale Stellung mit wesentlicher Sprechtempo- und Intensitätserhöhung. Phrasenartig bleibt beschleunigtes Sprechtempo noch bei wszystko. Bezug auf die Semantik kaum vorhanden.

PL 9

bardzo pomocne jest stosowanie formy (..) $\boldsymbol{m y}[\mathrm{i}::].(\mathrm{AI}++/ \mathrm{LS}+)($..) co wszyscy tutaj ... wiemy

Kommentar: Starke Segmentausdehnung verbunden sogar mit leichter Artikulationsspannung begleitet durch Intensitäts- und Lautstärkeerhöhung. Präpausale Stellung (Mangel an Präpause erschwert die Wahrnehmung). Dennoch effektive informative Hervorhebung des $m y$-Pronomens.

\section{Schlussfolgerungen}

Die oben dargestellte Analyse lässt einige Schlüsse hinsichtlich der Präsenz und rhetorischer Funktion der Fokusakzente in gesprochenen wissenschaftlichen Vorträgen im Deutschen und im Polnischen - allerdings nur vorsichtig - formulieren:

a. In gesprochenen Expertenvorträgen sind Fokusakzente relativ reichlich zu finden. Am häufigsten werden damit Einzelwörter (oft zusammengesetzte) hervorgehoben, seltener betrifft die Fokussierung kurze Phrasen. ${ }^{15}$

b. Es sind im analysierten Subkorpus keine wesentlichen - weder quantitative noch qualitative - Unterschiede zu finden, was die Verwendung der Fokusakzente im Deutschen und im Polnischen betrifft. Dies lässt schlussfolgern, dass sie kein sprachspezifisches Phänomen sind. Vielmehr können ihre Präsenz und Funktion idiolektal, kontextuell, situativ determiniert werden.

15 Im morphologischen bzw. syntaktischen Sinne. 
c. Fokusakzente werden in beiden Sprachen mit allen möglichen parametrisierbaren Realisierungsformen (Lautstärke, Sprechtempo, Intensität) prosodisch manifestiert.

d. Zur prosodischen Manifestation der Fokusakzente werden in den beiden Sprachen nicht selten artikulatorische Merkmale der Einzelsegmente ausgenutzt, indem sie modifiziert werden, ohne dass dabei ihr phonologischer Status irritiert wird (z.B. vokalische Quantitätsänderungen, Varianz der/r/-Aussprache).

e. Vereinzelt dient zum prosodischen Ausdruck der Fokusakzente in den beiden Sprachen die Verschiebung suprasegmentaler Wortbetonung.

f. Häufiger werden Fokusakzente internprosodisch kongruent markiert. Die Ausnutzung von nur einem Fokussierungsmarker kommt jedoch auch vor.

g. Man fokussiert nicht nur, indem bestimmte prosodische Parameter erhöht werden. Vereinzelt nutzt man auch ihre Senkung aus.

h. Externprosodisch kongruieren Fokusakzente in den beiden Sprachen sehr oft mit Pausen. Allem Anschein nach ist das Zusammenspiel von Fokusakzenten und Pausen ein besonders effektives Mittel prosodischer Hervorhebung.

i. Obwohl die meisten Fokusakzente zur Hervorhebung des informativ Signifikanten dienen, wird ihre Verwendung nicht immer semantisch determiniert. Vereinzelt können sie als syntaktische Referenzelemente gelten. Neben außerprosodischer sprachorganisatorischer Motivation beim Einsatz von Fokusakzenten ist auch die lautorganisatorische feststellbar.

j. In Bezug auf rhetorische Gestaltung eines gesprochenen wissenschaftlichen Vortrags können sowohl sprachorganisatorisch als auch lautorganisatorisch motivierte Fokusakzente ausgenutzt werden. Ars bene dicendi umfasst nämlich nicht nur entsprechende inhaltlich-informative Konstruktion einer Rede sondern auch ihre lautlich-rhythmisch-melodische Gestaltung. Als offen gelten jedoch im Zusammenhang damit unter anderen folgende Fragen: Wie sieht die Verteilung beider Schwerpunkte im Einzelnen aus? Ist sie völlig idiolektal/idiophonisch oder lässt sie sich einigermaßen systematisieren? Wie effektiv kann ihre Wahrnehmung sein?

Diese Fragen müssen als Postulate für weitere Forschung gelten. Die in den Punkten oben formulierten Erkenntnisse müssen unbedingt verifiziert werden, unter anderem indem die nötigen quantitativen Größen der untersuchten Texte in die Analysen miteinbezogen werden. Des Weiteren sind noch andere wissenschaftliche Textsorten und andere Sprachen zu involvieren, um ein möglicherweise wahres Bild von Verwendung phonetischer Mittel zur rhetorischen Gestaltung solcher Texte zu bekommen. 


\section{Literatur}

ALLNACH Konstanze / RUSCH Karoline, 1995, Rhetorik. Erfolgreiche Gesprächsführung, Redetechnik und Körpersprache mit Übungen und Musterreden, München.

Bose Ines / HiRSCHFELd Ursula / NeuBER Baldur / STOCK Eberhard, 2013, Einführung in die Sprechwissenschaft. Phonetik, Rhetorik, Sprechkunst, Tübingen.

CANEPARI Luciano, 2007, Natural Phonetics and Tonetics. Articulatory, Auditory, \& Functional, München.

FIUKOWSKI Hans, ${ }^{8} 2010$, Sprecherzieherisches Elementarbuch, Berlin/New York.

INOZUKA Emiko, 2003, Grundzüge der Intonation. Definition und Methodologie in deutschen Intonationsmodellen, Tübingen.

LEHISTE Ilse, 1970, Suprasegmentals, Cambridge (Mass.).

REINKE Kerstin, 2008, Zur Wirkung phonetischer Mittel in sachlich intendierter Sprechweise bei Deutsch sprechenden Russen, Frankfurt a. M.

SIKORSKI Janusz, 2003, Fonetyczny wykładnik gniewu. Uwagi z pogranicza kilku dyscyplin, in: Duszak A./Pawlak N. (Hrsg.), Anatomia gniewu. Emocje negatywne w językach i kulturach świata, Warszawa, S. 39-55.

TWOREK Artur, 2012, Einführung in die deutsch-polnische vergleichende Phonetik, Dresden/Wrocław.

TwOREK Artur, 2013, Subjekt-,Intonation“ vs. Objekt-,Intonation“, in: Błachut E./Gołębiowski A. (Hrsg.), Sprache in Wissenschaft und Unterricht (Akten der 23. intermnationalen Linguistenkonferenz Karpacz 14.-16.05.2012), Dresden/Wrocław, S. 149-158. 